\title{
Rockfall hazard assessment along a road on the Peloritani Mountains (northeastern Sicily, Italy)
}

\author{
G. Pappalardo ${ }^{1}$, S. Mineo ${ }^{2}$, and F. Rapisarda ${ }^{3}$ \\ ${ }^{1}$ Università degli Studi di Catania, Dipartimento di Scienze Biologiche Geologiche e Ambientali, Catania, Italy \\ ${ }^{2}$ Università degli Studi di Napoli Federico II, Dipartimento di Scienze della Terra dell'Ambiente e delle Risorse, Naples, Italy \\ ${ }^{3}$ Via Timone Zaccanazzo 4, Acireale, Catania, Italy \\ Correspondence to: S. Mineo (simomineo@gmail.com)
}

Received: 26 June 2013 - Published in Nat. Hazards Earth Syst. Sci. Discuss.: 6 December 2013

Revised: 9 September 2014 - Accepted: 10 September 2014 - Published: 10 October 2014

\begin{abstract}
A hazard assessment has been performed on rock slopes impending over a segment of the Taorminese Road, which connects two popular tourist destinations in northeastern Sicily: the urban centers of Taormina and Castelmola. The road crosses steep rock slopes with a complex geological and tectonic history. The section of the road close to Castelmola is often affected by rockfall phenomena, causing injury to people and serious damage to buildings and traffic. The study analyzes the geostructural setting of the unstable rock masses, by evaluating their mechanical properties and the kinematics of potential failures. Rockfall simulations confirm that falling rocks would involve the Taorminese Road with different kinetic energy rates and prove useful for suggesting the most suitable mitigation technologies for future remedial works. The modified Rockfall Hazard Rating System has been applied to highlight the different levels of hazard along the road. The compiled hazard map shows that portions of the slopes need urgent remedial works, especially because Taorminese is the only access road to Castelmola and its interruption would lead to the isolation of the village.
\end{abstract}

\section{Introduction}

Instability of rock slopes is a public safety issue, especially when its effects involve important communication routes. The economic impact of rockfalls on roads is considerable, because they often lead to traffic disruptions or delays and require expensive remedial measures (Turner and Schuster, 1996; Uribe-Etxebarria et al., 2005). When communication routes are located in mountain environments, it is also hard to find alternative viable ways. Therefore, rockfall has been a serious threat in many mountainous areas in the world (Chau et al., 2003; Dorren and Seijmonsbergen, 2003). In Italy, rockfalls represent a primary cause of landslide fatalities (Guzzetti et al., 2005; Palma et al., 2012).

The Taorminese Road (TR), a two-lane hill road from Taormina to Castelmola (6 km), northeastern Sicily (Fig. 1), is the case study of this paper. Taormina is a charming hillside town representing one of the most important tourist centers in Sicily; Castelmola, which probably used to be the Taormina acropolis, is a 1100-inhabitant village of preHellenic origin, counted among the Most Beautiful Italian Villages (Bacilieri, 2012). We have surveyed the TR segment closest to Castelmola $(1.7 \mathrm{~km}$ long; between $\mathrm{km} 6+100 \mathrm{~m}$ and $\mathrm{km} 7+800 \mathrm{~m}$ ), where several rockfall phenomena occurred in recent years, causing not only damage to man-made structures, but also isolating the village for prolonged periods (Ferrara and Pappalardo, 2005). Indeed, TR is the only access to Castelmola and the only escape route in case of evacuation. Its disruption would mean major setbacks not only from a touristic and economic point of view but also for rescue purposes. It crosses steep rock slopes, greatly affected by tectonics and intense fracturing. These factors, together with water infiltration and weathering, influence the stability of slopes (e.g., Crosta and Agliardi, 2003; Dorren and Seijmonsbergen, 2003; Jaboyedoff and Derron, 2005; Michoud et al., 2012). A few portions of the slopes, bordering the surveyed road segment, have wire meshes or retaining walls, while other unprotected areas are periodically subject to rockfalls, which represent a risk to vehicles and pedestrians. Indeed, the Italian Hydrogeological Basin Plan (P.A.I., 


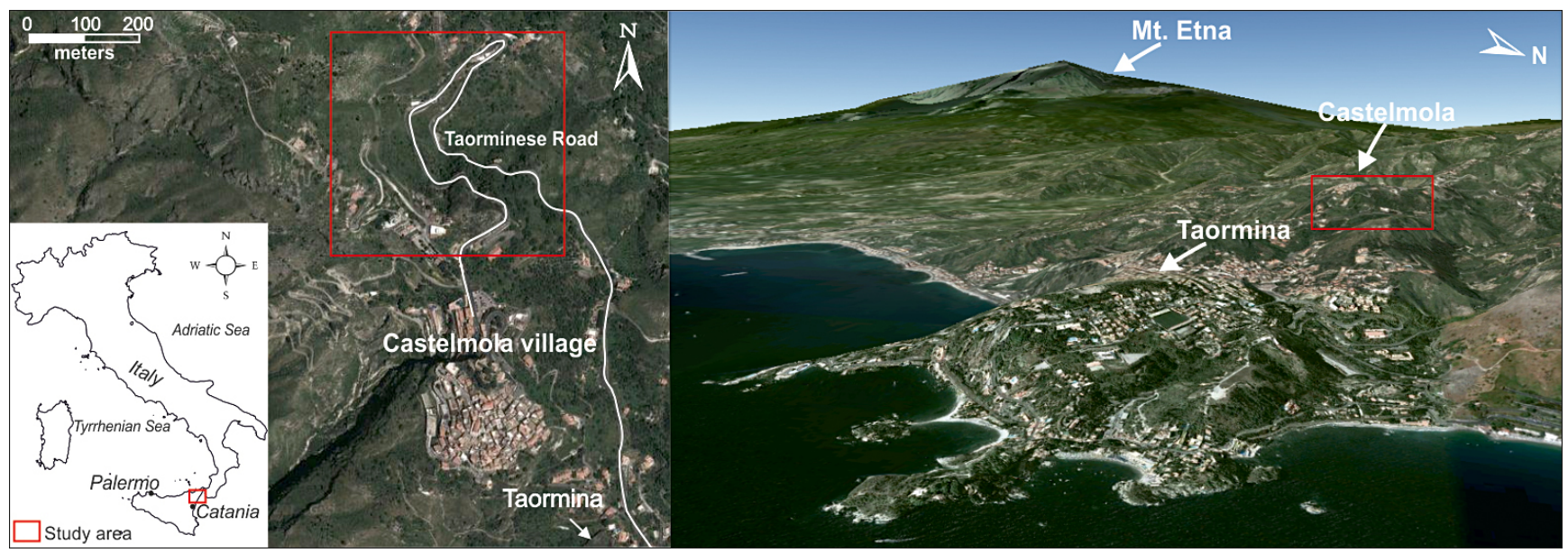

Figure 1. Geographical location of the study area.

2006), whose function is to identify high hazard and risk areas and to provide regulations and limitations for land use and development (Poretti and De Amicis, 2011), classifies the study area as a high rockfall risk zone.

This research deals with a rockfall hazard assessment carried out along the unprotected slopes, through the following steps: (1) collection of historical and recent rockfall information, (2) geostructural surveys, (3) rock and slope mass rating, (4) kinematic analysis, (5) two-dimensional rockfall trajectory simulations, and (6) application of the modified Rockfall Hazard Rating System (RHRS) (Pierson et al., 1990; Budetta, 2004).

\section{Geology and seismicity of the study area}

The study area lies in the Peloritani Mountain belt, which geologically represents the southern part of the CalabrianPeloritani Orogen (Atzori et al., 2003; Punturo et al., 2005; Cirrincione et al., 2012). It consists of a Hercynian metamorphic basement covered by Mesozoic-Cenozoic units (Punturo et al., 2005), displaced by regional fault systems, oriented W-E, NW-SE and NNW-SSE (Lentini et al., 2006). These structures are the main cause of the frequent seismic activity of the study area (Scandone et al., 1981; Tortorici et al., 1986), classified by the Italian Ordinance no. 3274/2003 as a category II area (medium-high seismic hazard) with peak ground acceleration of $0.25 \mathrm{~g}$. This is significant because the rockfall hazard is obviously higher in areas with intense seismic activity, where earthquakes are among the main triggering factors (Marinos and Tsiambaos, 2002; Saroglou et al., 2012; Barbano et al., 2014).

The stratigraphic succession of the study area (Fig. 2) is represented by a Hercynian crystalline basement (Paleozoic), overlain by sedimentary rocks belonging to the Taormina Unit (Catalano et al., 1995). The crystalline basement is represented by slates, belonging to a low metamorphic-grade complex with schistose texture, green-colored due to the presence of chlorite. The sedimentary cover consists of conglomerates and Triassic sandstones in Verrucano facies (Early Lias) (Dueé, 1969), greyish-white limestones and lower Liassic dolostones in carbonate platform facies (Lentini et al., 2006).

\section{Rockfall history}

Several rockfall phenomena have affected the whole territory of Castelmola, including the slopes impending over TR. Information on some of these landslides is available in the national databases of AVI (Italian Areas of Vulnerability; AVI Project, 1998) and P.A.I. (Piano stralcio per l'Assetto Idrogeologico, 2006). In 1952, the local daily newspaper $L a$ Gazzetta del Sud published an article on landslide movements occurring after heavy rain along the "only way of access to Castelmola". More recently, the main documented events, which interrupted TR near the access to the village, occurred in 1996, 1997 and 1999, when rockfalls from the northern cliff (Fig. 3), involving a significant volume of rock, occurred (Ferrara and Pappalardo, 2005). With respect to the 1999 event, the AVI database reports that a falling boulder involved a car, while no victims were reported. After these episodes, the northern cliff was consolidated by means of deep anchors, concrete retaining structures and drainage gullies at the base of the slope (Fig. 3).

In 2006, a boulder of about $6 \mathrm{~m}^{3}$ fell close to houses in the southeastern sector of the village (Fig. 3), prompting the municipal administration to perform urgent provisional works in order to install rockfall protection barriers behind the threatened houses.

In February 2012, two landslides occurred: the first overcame a retaining wall and invaded the road near a narrow curve; the second took place only $100 \mathrm{~m}$ away, destroying the wire mesh protecting the cliff (La Sicilia, 2012). During 


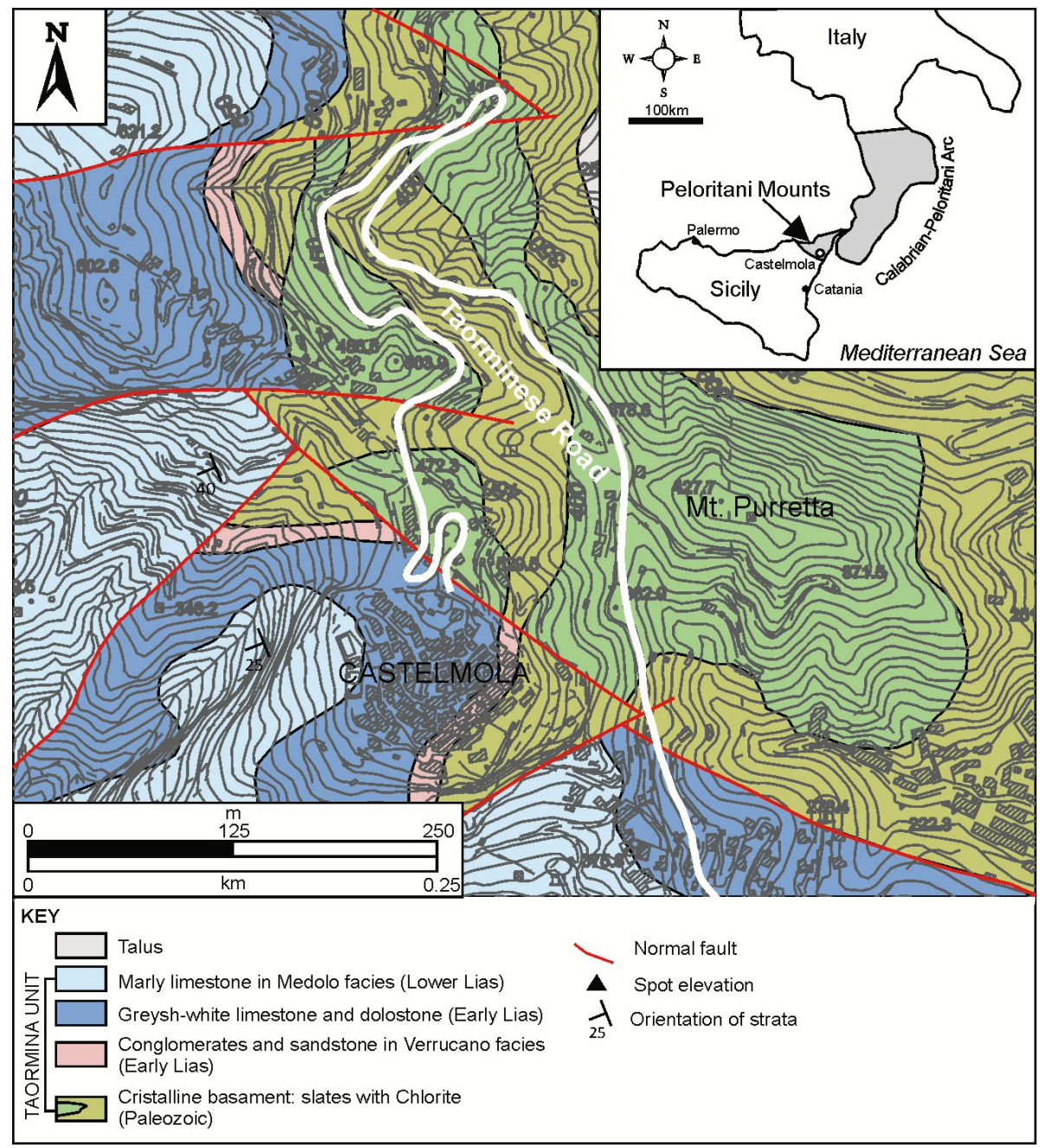

Figure 2. Geological setting of the study area. White line indicates the surveyed TR segment (modified after Carbone et al., 1994).

the night between 29 February 2012 and 1 March 2012, another landslide affected TR and threatened the water pipeline serving Taormina (available at: http://www.tempostretto.it, 2012).

However, since many events are not mentioned in the chronicles, most of the information regarding past rockfalls has been reported to us orally by local residents. In addition, during the surveys we noticed a number of boulders on the TR roadside (average size $0.5 \times 0.4 \times 0.5 \mathrm{~m}^{3}$ ), recently fallen and then moved away from the roadway (Fig. 3), as well as other boulders lying on the downstream slopes, evidencing how often rockfalls occur in this sector.

\section{Geostructural survey}

Geostructural surveys have been performed, following ISRM (2007) recommendations, in nine stations located on dolostone (D-St), limestone (L-St) and slate (S-St) outcrops. They were undertaken on slopes that were not fitted with rockfall protection measures and with evident unstable blocks that could be involved in rockfall phenomena.

The structural setting of dolostones is characterized by the presence of 4-5 intersecting discontinuity sets, whose spacing ranges from 2 to $60 \mathrm{~cm}$ and openings from 0.1 to $>5 \mathrm{~mm}$. Discontinuities are usually filled with sand or calcite and the joint surfaces are mostly smooth or undulated, with a joint roughness coefficient (JRC) (ISRM, 2007) ranging from 2 to 12 .

Within the limestone outcrops, four discontinuity sets are recognizable. These have a spacing generally ranging from 6 to $32 \mathrm{~cm}$, rarely with higher values that never exceed $100 \mathrm{~cm}$. Openings are often $>5 \mathrm{~mm}$ and filling is mostly absent in dry discontinuities, while it is soft or massive in wet ones. JRC is between 2 and 12. Small and micro caves due to carbonate chemical dissolution are present in the studied carbonate geological formation, while no considerable karst features have been surveyed in the outcrops of the study area. 

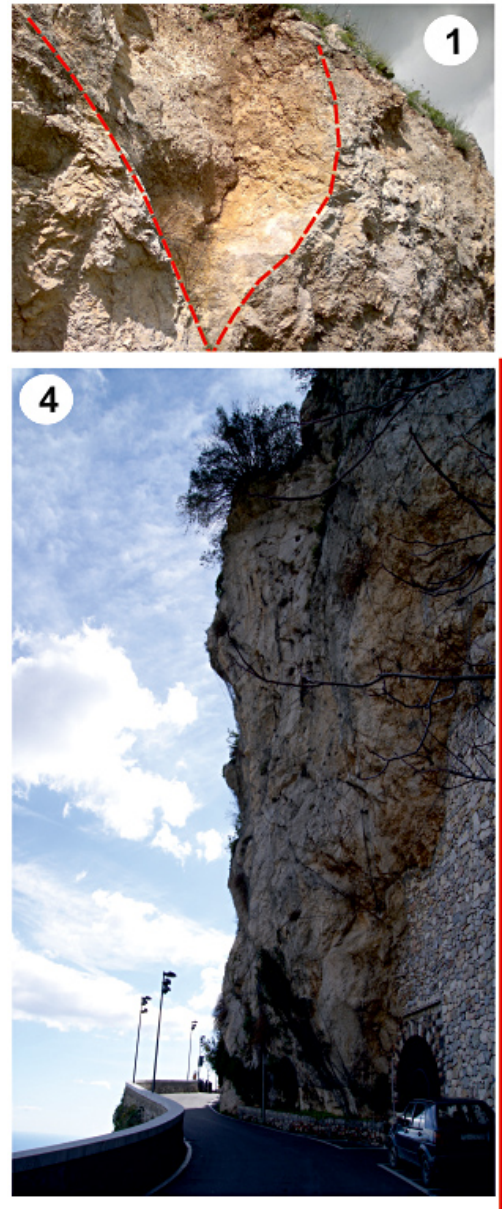
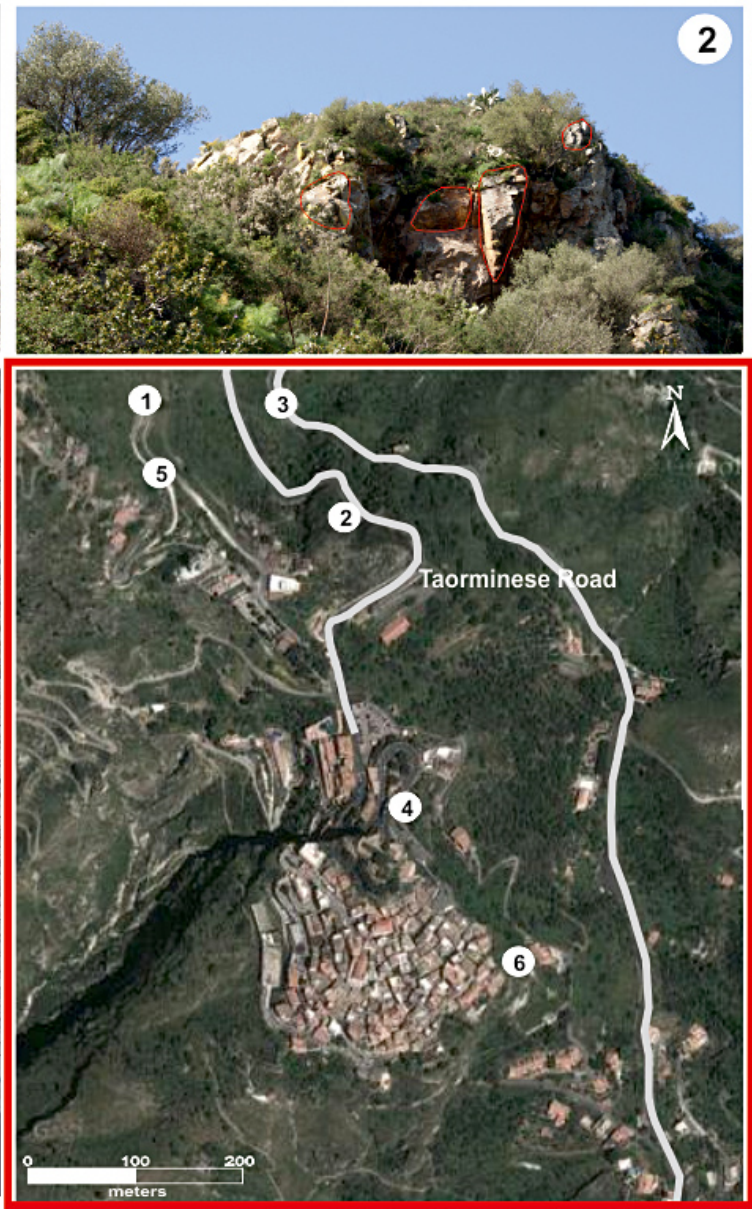
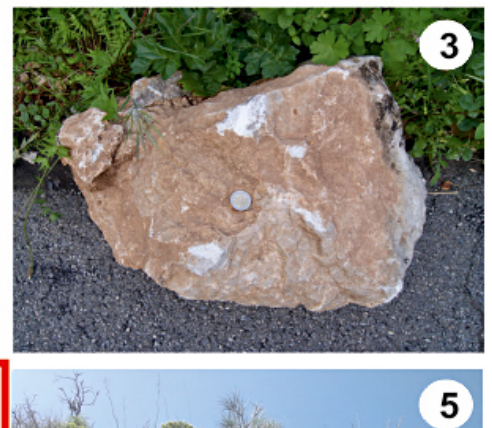

Figure 3. Example of unstable areas and occurred events. Key: 1, wedge sliding already occurred; 2, unstable blocks projecting on TR; 3 , one of the blocks found on the TR roadside; 4, northern cliff after remedial works; 5 , unstable wedge; and 6, boulder fallen in 2006 close to some houses.

Slate rock masses are pervaded by 3-4 discontinuity sets, with a $6-9 \mathrm{~cm}$ spacing, except for the F2 set $($ dip $/$ immersion $=336 / 8)$ whose spacing is about $150 \mathrm{~cm}$. Openings range from 0 to $>5 \mathrm{~mm}$ and no filling is present, while JRC ranges from 2 to 12 (Table 1).

Geomechanical characterization of the surveyed rock masses was performed (Table 2) in accordance with the classifications of Bieniawski (1989) and Romana (1985, 1988, $1991)$ in order to acquire information on the slope stability grade, the potential failure mode and the possible stabilization works. All the surveyed rock masses fall in the III Bieniawski class ("fair rock"), with $\mathrm{RMR}_{\mathrm{b}}$ (Rock Mass Rating) ranging between 42 and 58; and the mean cohesion values range between 211 and $288 \mathrm{KPa}$; mean internal friction angle varies from 26 to 34, according to the Bieniawski (1989) classification. With reference to the Romana (1985) classification, S-St-1 and D-St-3 are classified as "poor rock" (slope mass rating - SMR class IV); D-St-2 and D-St-4 as "very poor rock" (SMR class V). The others fall in III class ("fair rock").

\section{Kinematic analysis}

Kinematic analyses were performed using the Markland test (Markland, 1972), by analyzing the angular relationships between discontinuities and slope surfaces (Kliche, 1999), in order to determine the potential modes of failures among wedge sliding, planar siding and non-flexural toppling. Graphically, the great circle of a slope face and the friction angle circle $(\varphi)$ of the joint are plotted on a stereogram. The zone between the great circle and the friction circle is called "sliding envelope" (Yoon et al., 2002) and represents Markland's wedge and planar failure conditions (grey areas in Fig. 4). However, the condition for toppling failures occurs only if the layers strike parallel to the strike of the slope (Hoek and Bray, 1981; Adebimpe et al., 2011) but with an opposite immersion (red area in Fig. 4). The friction angle values considered here are those resulting from the Bieniawski (1989) rock mass classification discussed above (Table 2). 

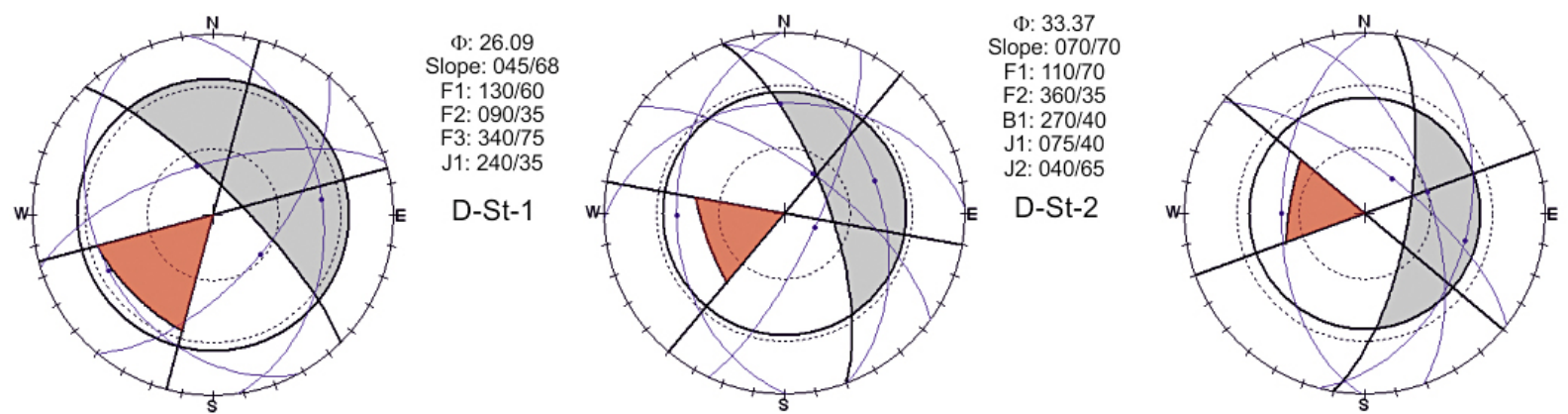

Ф: 36.89

Slope: 100/70

J3: $270 / 50$

B1: $270 / 50$

D-St-3

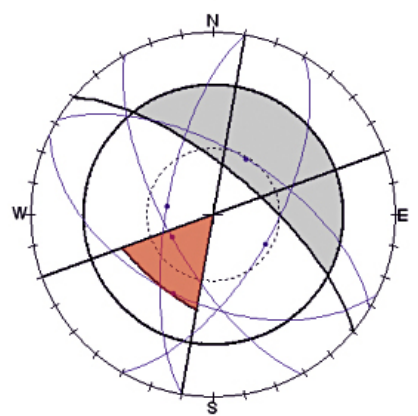

Ф: 29.56

Slope: 040/70

F1: 120/60

J1: $280 / 70$

J2: 030/60

J3: 240/70

B1: $210 / 55$
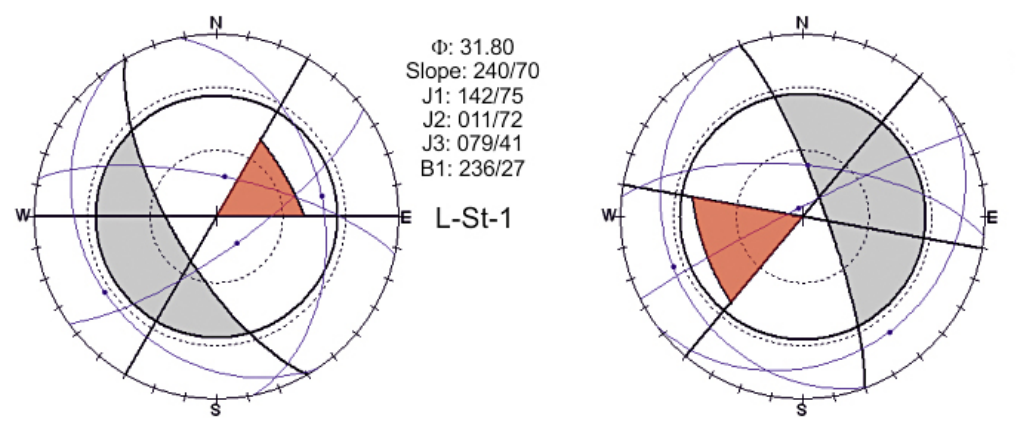

Ф: 33.78

Slope: $070 / 80$

J1: $143 / 42$

J2. $333 / 86$

J3: $006 / 67$

S1: $249 / 25$

D-St-4

L-St-2
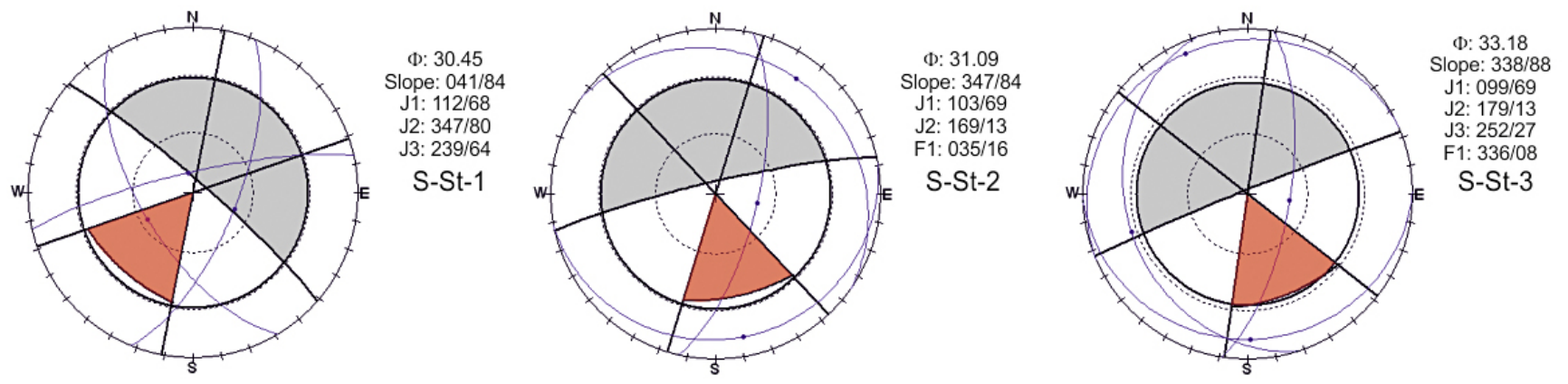

Figure 4. Markland test stereographic projections. Grey areas represent wedge and planar failure critical areas; red areas represent toppling failure critical areas.

With respect to the surveyed outcrops, the poorest stability conditions resulted within dolostones (D-St1, D-St2, D-St-3, D-St-4). The recognized modes of failure are (i) planar sliding, along discontinuities whose orientation is nearly parallel to the slope $\left( \pm 20^{\circ}\right)$, but with a lower inclination; (ii) wedge sliding, between two intersecting planes; and (iii) toppling, along discontinuities whose orientation is nearly parallel to the slope, but with an opposite immersion. Wedge sliding can occur at L-St-2; while on slates, wedge sliding and toppling are possible only at S-St-1 (Fig. 4).

\section{Rockfall analysis}

In order to assess if potential falling blocks could reach TR and to estimate their total kinetic energy, four rockfall simulations were performed. The chosen cross sections cut through the poorest quality rock masses with evident unstable blocks (Fig. 5). For simplicity, TR has been divided into upstream (UP) and downstream (DW) segments. The major difficulty in modeling the behavior of a rockfall event is characterizing all dependent variables thoroughly (De Almeida and Kullberg, 2011). Indeed, the relative movement of a falling boulder down a slope depends on a series of variable factors: the rock lithology, the topography and gradient of the slope (Parise, 2002) and the size and shape of the boulder (Schweigl et al., 2003). Consequently, it is certainly not a simple problem to forecast a rockfall trajectory accurately.

For the two-dimensional analysis of the motion of falling blocks, the calculation method proposed by Pfeiffer and Bowen (1989), who introduced it in the numerical code Colorado Rockfall Simulation Program (CRSP), has been chosen. The numerical code considers a single rock block as a simple point with a mass and a velocity (Massey et al., 2006) and, in order to describe the rockfall dynamics, it applies the equation of the parabolic motion of a free-falling mass and the principle of total energy conservation (Ferrero et al., 2011). Blocks can have spherical, cylindrical or discoid shape, with a circular section in the vertical plane of the 
Table 1. Surveyed geostructural parameters. JRC: joint roughness coefficient.

\begin{tabular}{|c|c|c|c|c|c|c|c|}
\hline $\begin{array}{l}\text { Set } \\
\text { system }\end{array}$ & $\begin{array}{l}\text { Spacing } \\
(\mathrm{mm})\end{array}$ & $\begin{array}{l}\text { Persistence } \\
(\mathrm{m})\end{array}$ & $\begin{array}{l}\text { Aperture } \\
(\mathrm{mm})\end{array}$ & Roughness & Infilling & Weathering & $\begin{array}{l}\text { Groundwater } \\
\text { condition }\end{array}$ \\
\hline \multicolumn{8}{|c|}{ D-St-1 (slope 045/68) } \\
\hline $\begin{array}{l}\text { F-1 } \\
(130 / 60)\end{array}$ & $\begin{array}{l}\text { Close } \\
(60-200)\end{array}$ & $\begin{array}{l}\text { High } \\
(10-20)\end{array}$ & $\begin{array}{l}\text { Moderate } \\
(2.5-10)\end{array}$ & $\begin{array}{l}\text { Smooth nearly planar } \\
(\mathrm{JRC}=2-4)\end{array}$ & $\begin{array}{l}\text { Soft } \\
>5 \mathrm{~mm}\end{array}$ & Moderate & Wet \\
\hline $\begin{array}{l}\text { F-2 } \\
(090 / 35)\end{array}$ & $\begin{array}{l}\text { Close } \\
(60-200)\end{array}$ & $\begin{array}{l}\text { High } \\
(10-20)\end{array}$ & $\begin{array}{l}\text { Moderate } \\
(2.5-10)\end{array}$ & $\begin{array}{l}\text { Undulating smooth to rough } \\
\text { (JRC }=8-12)\end{array}$ & $\begin{array}{l}\text { Soft } \\
>5 \mathrm{~mm}\end{array}$ & Moderate & Wet \\
\hline $\begin{array}{l}\text { F-3 } \\
(340 / 75)\end{array}$ & $\begin{array}{l}\text { Moderate } \\
(200-600)\end{array}$ & $\begin{array}{l}\text { High } \\
(10-20)\end{array}$ & $\begin{array}{l}\text { Moderate } \\
(2.5-10)\end{array}$ & $\begin{array}{l}\text { Undulating smooth to rough } \\
\text { (JRC }=8-12)\end{array}$ & $\begin{array}{l}\text { Hard } \\
>5 \mathrm{~mm}\end{array}$ & Moderate & Wet \\
\hline $\begin{array}{l}\mathrm{J}-1 \\
(240 / 35)\end{array}$ & $\begin{array}{l}\text { Moderate } \\
(200-600)\end{array}$ & $\begin{array}{l}\text { Low } \\
(1-3)\end{array}$ & $\begin{array}{l}\text { Partly open } \\
(0.25-0.5)\end{array}$ & $\begin{array}{l}\text { Undulating smooth to rough } \\
(\text { JRC }=8-12)\end{array}$ & None & Moderate & Wet \\
\hline \multicolumn{8}{|c|}{ D-St-2 (slope 070/70) } \\
\hline $\begin{array}{l}\text { F-1 } \\
(110 / 70)\end{array}$ & $\begin{array}{l}\text { Moderate } \\
(200-600)\end{array}$ & $\begin{array}{l}\text { High } \\
(10-20)\end{array}$ & $\begin{array}{l}\text { Moderate } \\
(2.5-10)\end{array}$ & $\begin{array}{l}\text { Undulating smooth to rough } \\
(\mathrm{JRC}=8-12)\end{array}$ & $\begin{array}{l}\text { Hard } \\
<5 \mathrm{~mm}\end{array}$ & Moderate & Wet \\
\hline $\begin{array}{l}\text { F-2 } \\
(360 / 35)\end{array}$ & $\begin{array}{l}\text { Close } \\
(60-200)\end{array}$ & $\begin{array}{l}\text { Very high } \\
(>20)\end{array}$ & $\begin{array}{l}\text { Very tight } \\
(<0.1)\end{array}$ & $\begin{array}{l}\text { Smooth nearly planar } \\
(\mathrm{JRC}=2-4)\end{array}$ & None & Slight & Dry \\
\hline $\begin{array}{l}\text { B-1 } \\
(270 / 40)\end{array}$ & $\begin{array}{l}\text { Moderate } \\
(200-600)\end{array}$ & $\begin{array}{l}\text { Very high } \\
(>20)\end{array}$ & $\begin{array}{l}\text { Moderate } \\
(2.5-10)\end{array}$ & $\begin{array}{l}\text { Undulating smooth to rough } \\
(\text { JRC }=8-12)\end{array}$ & $\begin{array}{l}\text { Hard } \\
>5 \mathrm{~mm}\end{array}$ & Slight & Dry \\
\hline $\begin{array}{l}\mathrm{J}-1 \\
(075 / 40)\end{array}$ & $\begin{array}{l}\text { Wide } \\
(600-2000)\end{array}$ & $\begin{array}{l}\text { Low } \\
(1-3)\end{array}$ & $\begin{array}{l}\text { Moderate } \\
(2.5-10)\end{array}$ & $\begin{array}{l}\text { Smooth nearly planar } \\
(\mathrm{JRC}=2-4)\end{array}$ & $\begin{array}{l}\text { Hard } \\
>5 \mathrm{~mm}\end{array}$ & Slight & Damp \\
\hline $\begin{array}{l}\mathrm{J}-2 \\
(040 / 65)\end{array}$ & $\begin{array}{l}\text { Wide } \\
(600-2000)\end{array}$ & $\begin{array}{l}\text { Low } \\
(1-3)\end{array}$ & $\begin{array}{l}\text { Partly open } \\
(0.25-0.5)\end{array}$ & $\begin{array}{l}\text { Smooth nearly planar } \\
(\mathrm{JRC}=2-4)\end{array}$ & None & Slight & Dry \\
\hline \multicolumn{8}{|c|}{ D-St-3 (slope 100/70) } \\
\hline $\begin{array}{l}\mathrm{J}-1 \\
(110 / 40)\end{array}$ & $\begin{array}{l}\text { Wide } \\
(600-2000)\end{array}$ & $\begin{array}{l}\text { Medium } \\
(3-10)\end{array}$ & $\begin{array}{l}\text { Partly open } \\
(0.25-0.5)\end{array}$ & $\begin{array}{l}\text { Undulating smooth to rough } \\
\text { (JRC }=8-12)\end{array}$ & None & Moderate & Damp \\
\hline $\begin{array}{l}\mathrm{J}-2 \\
(040 / 70)\end{array}$ & $\begin{array}{l}\text { Close } \\
(60-200)\end{array}$ & $\begin{array}{l}\text { Low } \\
(1-3)\end{array}$ & $\begin{array}{l}\text { Partly open } \\
(0.25-0.5)\end{array}$ & $\begin{array}{l}\text { Undulating smooth to rough } \\
\text { (JRC }=8-12)\end{array}$ & None & Slight & Damp \\
\hline $\begin{array}{l}\text { B-1 } \\
(270 / 50)\end{array}$ & $\begin{array}{l}\text { Wide } \\
(600-2000)\end{array}$ & $\begin{array}{l}\text { High } \\
(10-20)\end{array}$ & $\begin{array}{l}\text { Very tight } \\
(<0.1)\end{array}$ & $\begin{array}{l}\text { Undulating very rough } \\
(\mathrm{JRC}=16-18)\end{array}$ & None & Slight & Dry \\
\hline $\begin{array}{l}J-3 \\
(075 / 60)\end{array}$ & $\begin{array}{l}\text { Moderate } \\
(200-600)\end{array}$ & $\begin{array}{l}\text { Low } \\
(1-3)\end{array}$ & $\begin{array}{l}\text { Very tight } \\
(<0.1)\end{array}$ & $\begin{array}{l}\text { Undulating smooth to rough } \\
(\text { JRC }=8-12)\end{array}$ & None & Slight & Dry \\
\hline \multicolumn{8}{|c|}{ D-St-4 (slope 040/70) } \\
\hline $\begin{array}{l}\text { F-1 } \\
(120 / 60)\end{array}$ & $\begin{array}{l}\text { Very close } \\
(20-60)\end{array}$ & $\begin{array}{l}\text { High } \\
(10-20)\end{array}$ & $\begin{array}{l}\text { Partly open } \\
(0.25-0.5)\end{array}$ & $\begin{array}{l}\text { Smooth nearly planar } \\
(\mathrm{JRC}=2-4)\end{array}$ & None & Moderate & Dripping \\
\hline $\begin{array}{l}\text { J-1 } \\
(280 / 70)\end{array}$ & $\begin{array}{l}\text { Moderate } \\
(200-600)\end{array}$ & $\begin{array}{l}\text { Medium } \\
(3-10)\end{array}$ & $\begin{array}{l}\text { Moderate } \\
(2.5-10)\end{array}$ & $\begin{array}{l}\text { Undulating smooth to rough } \\
(\text { JRC }=8-12)\end{array}$ & $\begin{array}{l}\text { Hard } \\
>5 \mathrm{~mm}\end{array}$ & Moderate & Wet \\
\hline $\begin{array}{l}\mathrm{J}-2 \\
(030 / 60)\end{array}$ & $\begin{array}{l}\text { Close } \\
(60-200)\end{array}$ & $\begin{array}{l}\text { Low } \\
(1-3)\end{array}$ & $\begin{array}{l}\text { Partly open } \\
(0.25-0.5)\end{array}$ & $\begin{array}{l}\text { Smooth nearly planar } \\
(\mathrm{JRC}=2-4)\end{array}$ & None & Slight & Dripping \\
\hline $\begin{array}{l}\text { B-1 } \\
(210 / 55)\end{array}$ & $\begin{array}{l}\text { Moderate } \\
(200-600)\end{array}$ & $\begin{array}{l}\text { Low } \\
(1-3)\end{array}$ & $\begin{array}{l}\text { Very tight } \\
(<0.1)\end{array}$ & $\begin{array}{l}\text { Smooth nearly planar } \\
(\mathrm{JRC}=2-4)\end{array}$ & None & Slight & Damp \\
\hline $\begin{array}{l}\mathrm{J}-3 \\
(240 / 70)\end{array}$ & $\begin{array}{l}\text { Moderate } \\
(200-600)\end{array}$ & $\begin{array}{l}\text { Low } \\
(1-3)\end{array}$ & $\begin{array}{l}\text { Partly open } \\
(0.25-0.5)\end{array}$ & $\begin{array}{l}\text { Smooth nearly planar } \\
(\mathrm{JRC}=2-4)\end{array}$ & None & Slight & Damp \\
\hline
\end{tabular}


Table 1. Continued.

\begin{tabular}{|c|c|c|c|c|c|c|c|}
\hline $\begin{array}{l}\text { Set } \\
\text { system }\end{array}$ & $\begin{array}{l}\text { Spacing } \\
(\mathrm{mm})\end{array}$ & $\begin{array}{l}\text { Persistence } \\
(\mathrm{m})\end{array}$ & $\begin{array}{l}\text { Aperture } \\
(\mathrm{mm})\end{array}$ & Roughness & Infilling & Weathering & $\begin{array}{l}\text { Groundwater } \\
\text { condition }\end{array}$ \\
\hline \multicolumn{8}{|c|}{ L-St-1 (slope 240/70) } \\
\hline $\begin{array}{l}J-1 \\
(142 / 75)\end{array}$ & $\begin{array}{l}\text { Moderate } \\
(200-600)\end{array}$ & $\begin{array}{l}\text { Low } \\
(1-3)\end{array}$ & $\begin{array}{l}\text { Moderate } \\
(2.5-10)\end{array}$ & $\begin{array}{l}\text { Undulating smooth to rough } \\
\text { (JRC }=8-12)\end{array}$ & None & Moderate & Dry \\
\hline $\begin{array}{l}\mathrm{J}-2 \\
(11 / 72)\end{array}$ & $\begin{array}{l}\text { Moderate } \\
(200-600)\end{array}$ & $\begin{array}{l}\text { Low } \\
(1-3)\end{array}$ & $\begin{array}{l}\text { Moderate } \\
(2.5-10)\end{array}$ & $\begin{array}{l}\text { Undulating smooth to rough } \\
(\text { JRC }=8-12)\end{array}$ & $\begin{array}{l}\text { Hard } \\
>5 \mathrm{~mm}\end{array}$ & Moderate & Dry \\
\hline $\begin{array}{l}\mathrm{J}-3 \\
(79 / 41)\end{array}$ & $\begin{array}{l}\text { Moderate } \\
(200-600)\end{array}$ & $\begin{array}{l}\text { Medium } \\
(3-10)\end{array}$ & $\begin{array}{l}\text { Moderate } \\
(2.5-10)\end{array}$ & $\begin{array}{l}\text { Smooth nearly planar } \\
(\text { JRC }=2-4)\end{array}$ & $\begin{array}{l}\text { Hard } \\
>5 \mathrm{~mm}\end{array}$ & Slight & Dry \\
\hline $\begin{array}{l}\text { B-1 } \\
(236 / 27)\end{array}$ & $\begin{array}{l}\text { Moderate } \\
(200-600)\end{array}$ & $\begin{array}{l}\text { Very high } \\
(>20)\end{array}$ & $\begin{array}{l}\text { Moderate } \\
(2.5-10)\end{array}$ & $\begin{array}{l}\text { Undulating smooth to rough } \\
(\mathrm{JRC}=8-12)\end{array}$ & None & Slight & Dry \\
\hline \multicolumn{8}{|c|}{ L-St-2 (slope 070/80) } \\
\hline $\begin{array}{l}\mathrm{J}-1 \\
(143 / 42)\end{array}$ & $\begin{array}{l}\text { Wide } \\
(600-2000)\end{array}$ & $\begin{array}{l}\text { High } \\
(10-20)\end{array}$ & $\begin{array}{l}\text { Moderate } \\
(2.5-10)\end{array}$ & $\begin{array}{l}\text { Smooth nearly planar } \\
(\text { JRC }=2-4)\end{array}$ & $\begin{array}{l}\text { Hard } \\
>5 \mathrm{~mm}\end{array}$ & Slight & Damp \\
\hline $\begin{array}{l}\mathrm{J}-2 \\
(333 / 86)\end{array}$ & $\begin{array}{l}\text { Moderate } \\
(200-600)\end{array}$ & $\begin{array}{l}\text { Medium } \\
(3-10)\end{array}$ & $\begin{array}{l}\text { Moderate } \\
(2.5-10)\end{array}$ & $\begin{array}{l}\text { Undulating smooth to rough } \\
(\mathrm{JRC}=8-12)\end{array}$ & $\begin{array}{l}\text { Soft } \\
>5 \mathrm{~mm}\end{array}$ & Moderate & Damp \\
\hline $\begin{array}{l}J-3 \\
(6 / 67)\end{array}$ & $\begin{array}{l}\text { Moderate } \\
(200-600)\end{array}$ & $\begin{array}{l}\text { Medium } \\
(3-10)\end{array}$ & $\begin{array}{l}\text { Moderate } \\
(2.5-10)\end{array}$ & $\begin{array}{l}\text { Smooth nearly planar } \\
(\text { JRC }=2-4)\end{array}$ & None & Slight & Dry \\
\hline $\begin{array}{l}\text { S-1 } \\
(249 / 25)\end{array}$ & $\begin{array}{l}\text { Very close } \\
(20-60)\end{array}$ & $\begin{array}{l}\text { Very high } \\
(>20)\end{array}$ & $\begin{array}{l}\text { Moderate } \\
(2.5-10)\end{array}$ & $\begin{array}{l}\text { Smooth nearly planar } \\
(\mathrm{JRC}=2-4)\end{array}$ & None & Moderate & Dry \\
\hline \multicolumn{8}{|c|}{ S-St-1 (slope 041/84) } \\
\hline $\begin{array}{l}J-1 \\
(112 / 68)\end{array}$ & $\begin{array}{l}\text { Very close } \\
(20-60)\end{array}$ & $\begin{array}{l}\text { High } \\
(10-20)\end{array}$ & $\begin{array}{l}\text { Moderate } \\
(2.5-10)\end{array}$ & $\begin{array}{l}\text { Smooth nearly planar } \\
(\mathrm{JRC}=2-4)\end{array}$ & $\begin{array}{l}\text { Soft } \\
<5 \mathrm{~mm}\end{array}$ & Moderate & Damp \\
\hline $\begin{array}{l}J-2 \\
(347 / 80)\end{array}$ & $\begin{array}{l}\text { Ext. close } \\
(<20)\end{array}$ & $\begin{array}{l}\text { High } \\
(10-20)\end{array}$ & $\begin{array}{l}\text { Moderate } \\
(2.5-10)\end{array}$ & $\begin{array}{l}\text { Smooth nearly planar } \\
(\text { JRC }=2-4)\end{array}$ & $\begin{array}{l}\text { Soft } \\
<5 \mathrm{~mm}\end{array}$ & Moderate & Damp \\
\hline $\begin{array}{l}J-3 \\
(239 / 64)\end{array}$ & $\begin{array}{l}\text { Wide } \\
(600-2000)\end{array}$ & $\begin{array}{l}\text { Medium } \\
(3-10)\end{array}$ & $\begin{array}{l}\text { Moderate } \\
(2.5-10)\end{array}$ & $\begin{array}{l}\text { Undulating smooth to rough } \\
(\mathrm{JRC}=8-12)\end{array}$ & $\begin{array}{l}\text { Hard } \\
<5 \mathrm{~mm}\end{array}$ & Slight & Dry \\
\hline \multicolumn{8}{|c|}{ S-St-2 (slope 347/84) } \\
\hline $\begin{array}{l}J-1 \\
(103 / 69)\end{array}$ & $\begin{array}{l}\text { Moderate } \\
(200-600)\end{array}$ & $\begin{array}{l}\text { High } \\
(10-20)\end{array}$ & $\begin{array}{l}\text { Moderate } \\
(2.5-10)\end{array}$ & $\begin{array}{l}\text { Smooth nearly planar } \\
(\mathrm{JRC}=2-4)\end{array}$ & None & Moderate & Damp \\
\hline $\begin{array}{l}\mathrm{J}-2 \\
(169 / 13)\end{array}$ & $\begin{array}{l}\text { Close } \\
(60-200)\end{array}$ & $\begin{array}{l}\text { Medium } \\
(3-10)\end{array}$ & $\begin{array}{l}\text { Very tight } \\
(<0.1)\end{array}$ & $\begin{array}{l}\text { Slickensided planar } \\
(\mathrm{JRC}=0-2)\end{array}$ & None & Slight & Dry \\
\hline $\begin{array}{l}\text { F-1 } \\
(35 / 16)\end{array}$ & $\begin{array}{l}\text { Ext. close } \\
(<20)\end{array}$ & $\begin{array}{l}\text { High } \\
(10-20)\end{array}$ & $\begin{array}{l}\text { Moderate } \\
(2.5-10)\end{array}$ & $\begin{array}{l}\text { Smooth nearly planar } \\
(\mathrm{JRC}=2-4)\end{array}$ & $\begin{array}{l}\text { Soft } \\
>5 \mathrm{~mm}\end{array}$ & High & Damp \\
\hline \multicolumn{8}{|c|}{ S-St-3 (slope 338/88) } \\
\hline $\begin{array}{l}J-1 \\
(99 / 69)\end{array}$ & $\begin{array}{l}\text { Close } \\
(60-200)\end{array}$ & $\begin{array}{l}\text { High } \\
(10-20)\end{array}$ & $\begin{array}{l}\text { Moderate } \\
(2.5-10)\end{array}$ & $\begin{array}{l}\text { Smooth nearly planar } \\
(\mathrm{JRC}=2-4)\end{array}$ & None & Moderate & Damp \\
\hline $\begin{array}{l}\mathrm{J}-2 \\
(179 / 13)\end{array}$ & $\begin{array}{l}\text { Ext. close } \\
(<20)\end{array}$ & $\begin{array}{l}\text { Low } \\
(1-3)\end{array}$ & $\begin{array}{l}\text { Very tight } \\
(<0.1)\end{array}$ & $\begin{array}{l}\text { Slickensided planar } \\
(\mathrm{JRC}=0-2)\end{array}$ & None & Moderate & Dry \\
\hline $\begin{array}{l}\mathrm{J}-3 \\
(252 / 27)\end{array}$ & $\begin{array}{l}\text { Close } \\
(60-200)\end{array}$ & $\begin{array}{l}\text { Low } \\
(1-3)\end{array}$ & $\begin{array}{l}\text { Very tight } \\
(<0.1)\end{array}$ & $\begin{array}{l}\text { Smooth nearly planar } \\
(\text { JRC }=2-4)\end{array}$ & None & Moderate & Dry \\
\hline $\begin{array}{l}\text { F-1 } \\
(336 / 8)\end{array}$ & $\begin{array}{l}\text { Wide } \\
(600-2000)\end{array}$ & $\begin{array}{l}\text { High } \\
(10-20)\end{array}$ & $\begin{array}{l}\text { Moderate } \\
(2.5-10)\end{array}$ & $\begin{array}{l}\text { Smooth nearly planar } \\
(\text { JRC }=2-4)\end{array}$ & $\begin{array}{l}\text { Soft } \\
>5 \mathrm{~mm}\end{array}$ & High & Damp \\
\hline
\end{tabular}


Table 2. Rock masse classification parameters.

\begin{tabular}{lcccccl}
\hline $\begin{array}{l}\text { Survey } \\
\text { station }\end{array}$ & RMR $_{\mathrm{b}}$ & $\begin{array}{c}\text { Bieniawski } \\
\text { (1989) class }\end{array}$ & $\begin{array}{c}c^{\prime} \\
(\mathrm{KPa})\end{array}$ & $\begin{array}{c}\Phi^{\prime} \\
\left(^{\circ}\right)\end{array}$ & SMR & $\begin{array}{l}\text { Romana (1985) } \\
\text { class }\end{array}$ \\
\hline D-St-1 & 42 & III (fair rock) & 211 & 26 & 43 & III (fair rock) \\
D-St-2 & 57 & III (fair rock) & 284 & 33 & 13 & V (very poor) \\
D-St-3 & 54 & III (fair rock) & 269 & 32 & 22 & IV (poor rock) \\
D-St-4 & 49 & III (fair rock) & 246 & 30 & 19 & V (very poor) \\
L-St-1 & 54 & III (fair rock) & 268 & 32 & 42 & III (fair rock) \\
L-St-2 & 58 & III (fair rock) & 288 & 34 & 50 & III (fair rock) \\
S-St-1 & 51 & III (fair rock) & 254 & 30 & 36 & IV (poor rock) \\
S-St-2 & 52 & III (fair rock) & 261 & 31 & 54 & III (fair rock) \\
S-St-3 & 56 & III (fair rock) & 282 & 33 & 59 & III (fair rock) \\
\hline
\end{tabular}

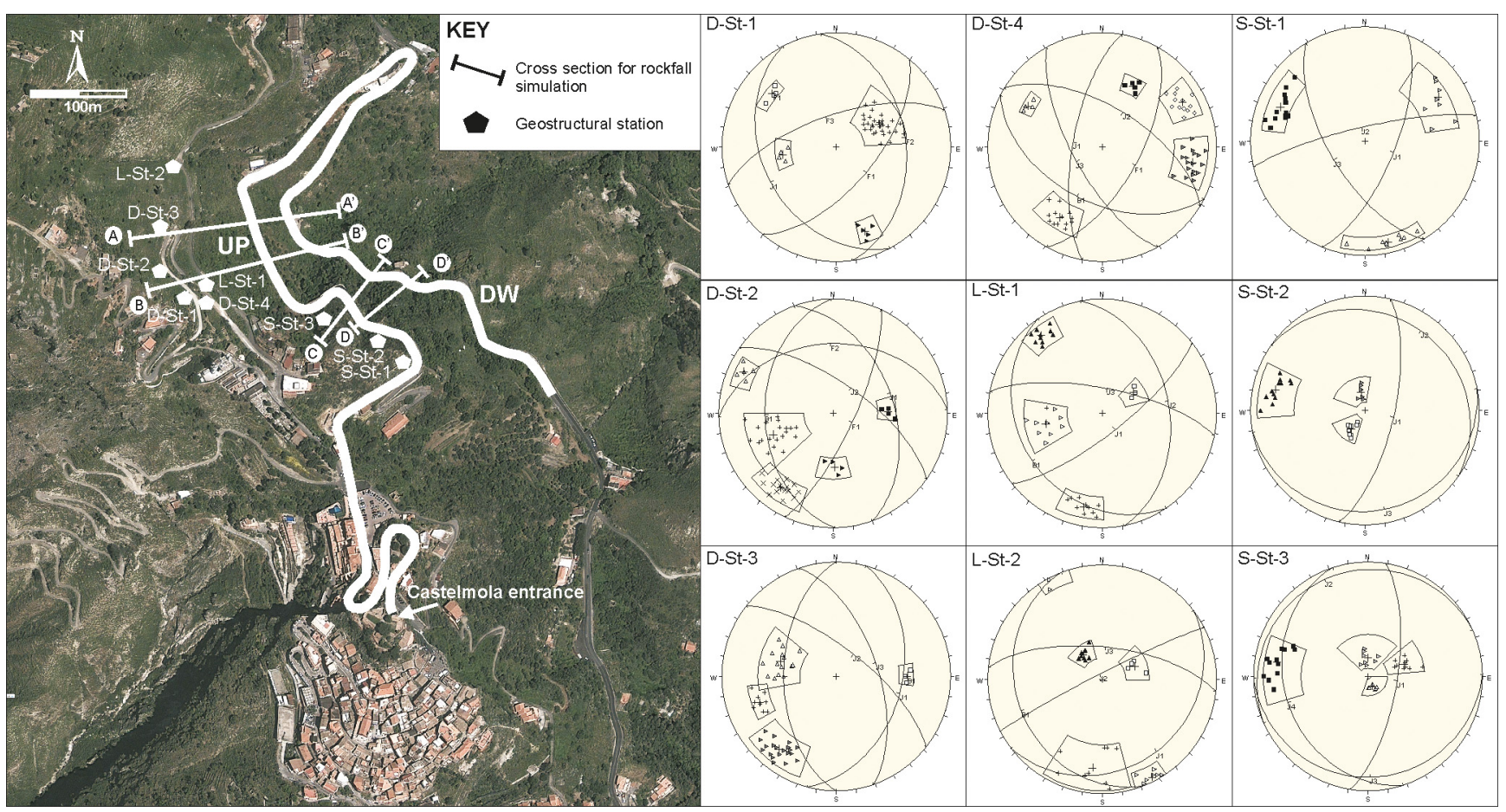

Figure 5. Cross sections for rockfall simulation and location of geostructural survey stations with resulting stereonets.

motion. The analysis considers the combined effects of free falling, bouncing, rolling and sliding; the block impact is influenced by the slope roughness and the block size (Giani, 1992).

With respect to the block size, a $250 \mathrm{~kg}$ boulder $\left(0.5 \times 0.4 \times 0.5 \mathrm{~m}^{3}\right)$ has been considered, similar to the ones recently found on the Taorminese roadside (Fig. 3). Its initial velocity, although Paronuzzi (1987a) and Azzoni et al. (1995) consider it negligible because of its very low value, was here estimated by taking into account the seismic conditions of the area, according to Antoniou and Lekkas (2010), through Eq. (1):

$v=\sqrt{2 a s}$, where $a$ is the ground acceleration, here assumed as $0.25 \mathrm{~g}$ (according to the Italian Ordinance no. 3274/2003), and $s$ is the distance between the rock fragment and the slope, produced by the seismic wave action (Antoniou and Lekkas, 2010).

The most difficult variables to define in this type of analysis are the coefficients of restitution $\left(R_{\mathrm{n}}\right.$ : normal; and $R_{\mathrm{t}}$ : tangential) of the slope materials (Richards et al., 2001; Asteriou et al., 2012), strongly influenced by the impact conditions (Paronuzzi, 2009). Both parameters are determined by the composition and size of the material covering the surface and the radius of the falling block itself (Dorren et al., 2006).

The events occurring in the study area have never been studied before and all their traces have been obliterated by 

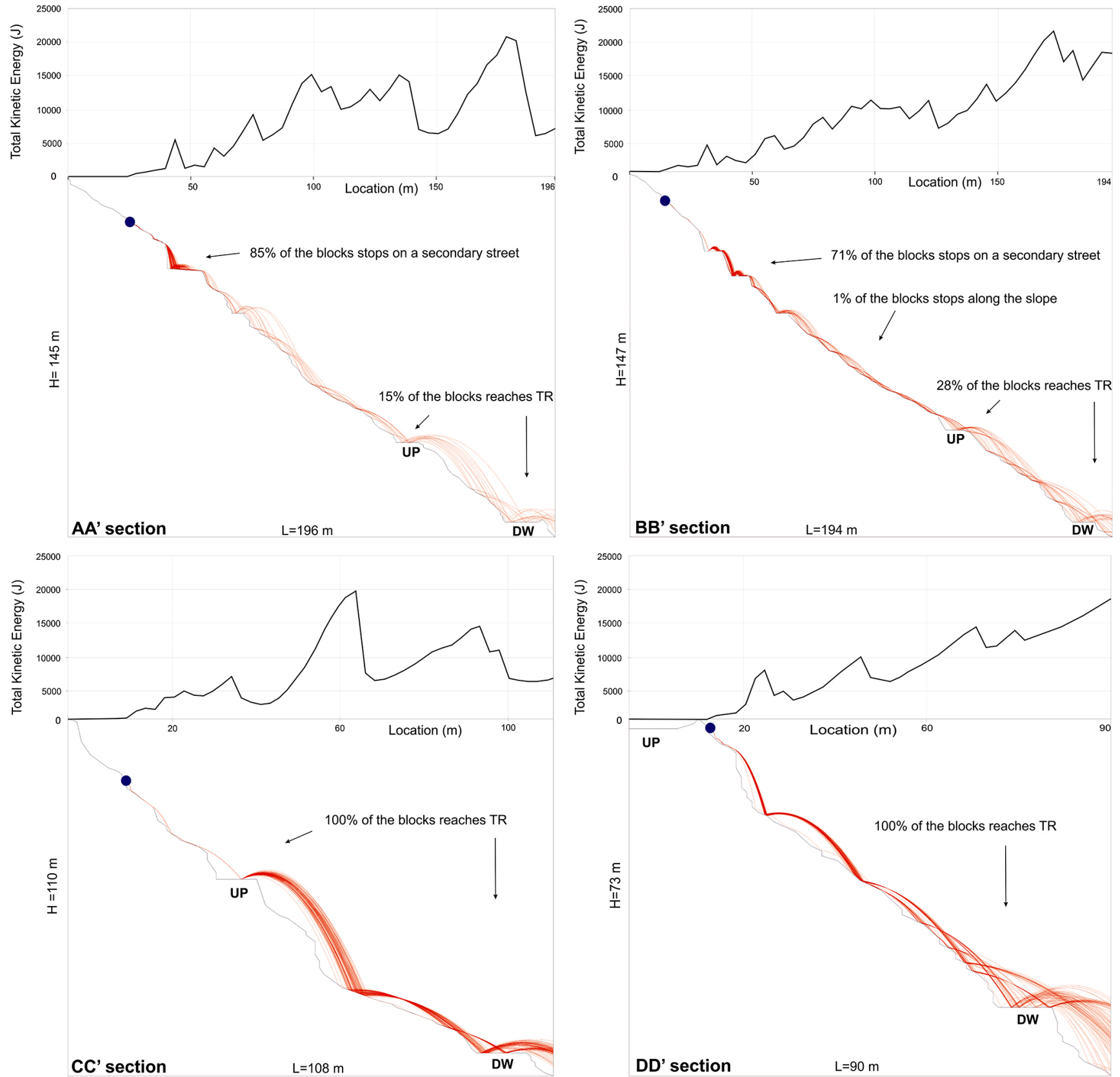

Figure 6. Rockfall simulations and kinetic energy envelope. The blue circles indicate the detachment points.

time and human activity. Therefore, in order to find the most suitable coefficient for the surveyed slopes, we have taken into account both a back analysis performed in a neighboring area with similar geological features, located at the south-eastern sector of the Castelmola cliff, and the coefficients of restitution retrieved from the published literature (e.g., Pfeiffer and Bowen, 1989; Giani, 1992; Robotham et al., 1995; Chau et al., 1998; Dorren and Seijmonsbergen, 2003; Massey et al., 2006; Budetta, 2010; Pantelidis and Kokkalis, 2011; Saroglou et al., 2012) (Table 3).
The simulation results show that in the $\mathrm{AA}^{\prime}$ and $\mathrm{BB}^{\prime}$ sections 85 and $71 \%$, respectively, of the falling rocks stop on a secondary road, which connects Castelmola to some private properties. The remaining 15 and $28 \%$ reach the TR UP segment and bounce onto the DW segment, which, however, does not represent the rockfalls end point. With respect to the BB' section, $1 \%$ of the blocks end their run along the slope, before reaching TR (Fig. 6). On the steepest slopes (CC' and DD' sections), $100 \%$ of the boulders initially impact on the 
Table 3. Coefficients of restitution applied for rockfall simulations.

\begin{tabular}{|c|c|c|c|}
\hline Authors & Material type & $R_{\mathrm{t}}$ & $R_{\mathrm{n}}$ \\
\hline \multirow[t]{2}{*}{ Crosta and Agliardi (2003) } & Outcropping rock, bare & $0.75 \pm 0.0150$ & $0.50 \pm 0.0125$ \\
\hline & Outcropping rock, forested & $0.70 \pm 0.0140$ & $0.50 \pm 0.01$ \\
\hline Schweigl et al. (2003) & Asphalt & $0.90 \pm 0.04$ & $0.40 \pm 0.04$ \\
\hline Hoek (1987) & Asphalt roadway & 0.90 & 0.40 \\
\hline Pfeiffer and Bowen (1989) & Bedrock or boulders with little soil or vegetation & $0.83-0.87$ & $0.33-0.37$ \\
\hline \multirow{3}{*}{$\begin{array}{l}\text { Neighboring back analysis } \\
\text { (southeastern sector of } \\
\text { Castelmola cliff) }\end{array}$} & Bare rock & 0.9 & 0.7 \\
\hline & Rock debris & 0.65 & 0.15 \\
\hline & Rock debris with vegetation and shrubs & 0.53 & 0.15 \\
\hline
\end{tabular}

UP segment, then, after bouncing one or two times along the slope, reach the DW segment (Fig. 6).

Blocks move along the slope rolling and bouncing, depending on the slope inclination and the presence of vegetation or bare rock. Bounce height at TR ranges from $<1$ to $6 \mathrm{~m}$ at UP and from $<1$ to $9 \mathrm{~m}$ at DW. Kinetic energy values vary with respect to the type of movement; indeed, lower energy can be related to rolling blocks, while higher rates are released at bouncing points. In particular, kinetic energy estimated on TR ranges between 1.2 and $20.2 \mathrm{~kJ}$ with a modal value of $8 \mathrm{~kJ}$.

\section{Rockfall hazard assessment}

The RHRS is a semi-quantitative classification system, developed by the Oregon Department of Transportation (USA), to assess the hazard associated with rockfalls (Pierson et al., 1990; National Highway Institute, 1993), in order to identify dangerous slopes that require urgent remedial works or further studies. The method was subsequently modified to make it more suitable to the geometrical features and to the traffic standards of the Italian roads (Budetta, 2004).

It consists in assigning a score to nine categories concerning the rockfall hazard (i.e., slope height, geological features, volume of rockfall/block size, climate and presence of water on slope and rockfall history) and the vehicle vulnerability (i.e., ditch effectiveness, average vehicle risk, percent of decision sight distance, and roadway width). The sum of all the assigned scores expresses the degree of exposure to the hazard along roads. If the RHRS final value is lower than 300 , the remedial works on the slope will be considered "with low urgency"; however, if the final score is higher than 500, the slope will need "immediate stabilization works" (Pierson et al., 1990). Slopes with intermediate scores are considered with "high priority of remedial works", although a case-bycase evaluation would be appropriate (Budetta, 2004).
In this paper, hazard has been assessed at 12 measure stations, located on slopes with no protection measures (between km 6+250 m-6 +500 m and km 6+900 m$7+300 \mathrm{~m}$ ) (Fig. 7), in both directions of travel. Each required parameter has been calculated by the equations proposed by Budetta (2004):

1. Slope heights are all greater than $30 \mathrm{~m}$.

2. Average vehicle risk (AVR) ranges from 3.75 to $10 \%$, with assigned scores between 1 and 2 depending on to the hazard zone length (speed limit $40 \mathrm{~km} \mathrm{~h}^{-1}$ ). AVR has been calculated considering a daily traffic of 2500 units (cars, motorcycles, bicycles and buses), estimated by traffic measurements conducted on a 2-day survey between 10:00 and 18:00 LT. This value includes a rate of tourist units, whose maximum peak is usually registered during summer and weekends, and the daily commuters traveling by car to and from Castelmola (Corriere and Russo, 2003).

3. Decision sight distance (DSD) ranges between 6.99 and $69.93 \%$, with minimum values measured close to the bends (reduced visibility) located in the DW segment. This parameter has the largest influence on the final score.

4. Slope mass rating values have been taken from the Romana classification performed on the surveyed rock masses (see Sect. 4).

5. Block size has been assumed as $50 \mathrm{~cm}$, similar to the ones recently found on the TR roadside (Fig. 3).

6. Mean annual rainfall value is related to the period 19212003 (900 $\mathrm{mm} \mathrm{yr}^{-1}$ ), according to the 1921-2003 map of isohyets (Assessorato Regionale per i rifiuti acque settore Osservatorio delle Acque, 2004). 


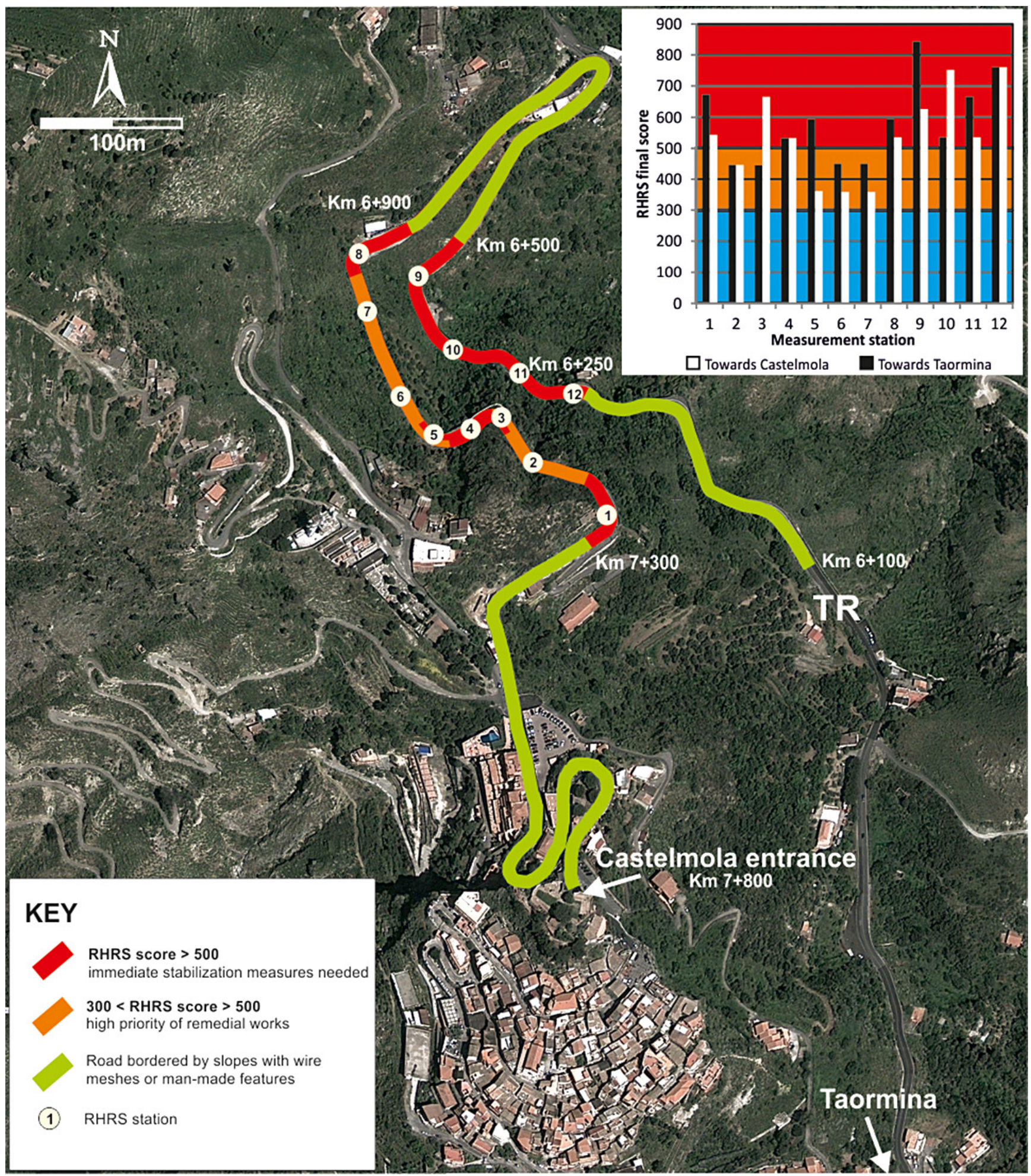

Figure 7. Taorminese Road hazard distribution. The histogram in the upper right corner shows the final RHRS scores for each measurement station. 
7. Six events per year have been assumed as the rockfall frequency, according to the information retrieved during our surveys.

The calculated final scores range between 359 and 844; the minimum value has been estimated at sections 6 and $7(\mathrm{~km} 7+60 \mathrm{~m}$ and $7+00 \mathrm{~m})$ toward Castelmola, while the maximum value at section $9(\mathrm{~km} 6+250 \mathrm{~m})$ toward Taormina (Fig. 7). According to these scores, the UP segment is classifiable as a road with "high priority of remedial works" $(300<$ RHRS $<500)$, although there are three bend portions with RHRS $>500$. The highest hazard has been detected at bend portions of UP and along the DW segment, where "immediate stabilization works" (RHRS > 500) would be needed. This is due to a higher number of bends along the DW segment than the UP segment, which causes considerable changes in DSD, leading to higher hazard at low-visibility points (bends).

\section{Conclusions}

Communication routes in mountainous areas are often affected by slope instability problems. A segment of the Taorminese Road (TR) has been studied with the aim of assessing the hazard connected to rockfall phenomena. TR is a road of strategic local importance because it is the only route connecting Taormina to Castelmola, two important tourist destinations in northeastern Sicily. The final segment of TR, which leads to Castelmola, has a long history of rockfalls that have isolated the entire village, hindering the local social and economic activities.

The performed study indicates that the complex geological history of the area and its seismicity contribute to slope instability. Indeed, the geological formations have been displaced, during several tectonic stages, by different fault systems. The effects are clearly visible on the outcrops and the geostructural study highlighted that several discontinuity sets pervade the rock masses, which have been classified as "fair", "poor" and "very poor" by the Bieniawski and Romana indexes. Kinematic analysis performed on nine survey stations recognized, among the potential failure modes, the possibility of planar and wedge sliding, as well as toppling. Small and micro karst caves seem not to play a significant role for the slope stability.

Rockfall simulations showed that falling boulders would reach both the upstream and the downstream segments of TR. It should be noted that, with respect to the considered sections, we have placed emphasis only on a $250 \mathrm{~kg}$ falling block, in accordance with the size of previously fallen boulders, without taking into account the mobilization of further material, as well as the possibility of block fragmentation upon impacts along the slope.

Hazard analysis was performed using the modified Rockfall Hazard Rating System (RHRS), which classified the road portions between $\mathrm{km} 6+250 \mathrm{~m}-6+500 \mathrm{~m}$ and $\mathrm{km} 6+900 \mathrm{~m}-7+300 \mathrm{~m}$ as a high rockfall hazard road. It is therefore clear that remedial works are needed along such portions in order to protect the road itself. Considering the high degree of rock mass fracturing and the possibility of occurring failures, the installation of wire meshes and deformable rockfall barriers would be appropriate. In particular, wire meshes should be affixed to vertical, bare slopes in order to contain block detachments, while rockfall barriers should be placed as a protection fence of the upstream segment.

Acknowledgements. The authors wish to thank the Editor Mario Parise, Haralambos Saroglou, Sebastiano Perriello Zampelli and all the anonymous referees who supported our work with their valuable suggestions.

Edited by: M. Parise

Reviewed by: H. Saroglou, S. Perriello Zampelli, and three anonymous referees

\section{References}

Adebimpe, R. A., Akande, J. M., and Arum, C.: Slope Stability Analysis of Itakpe Iron Ore Mine, Itakpe, Nigeria, Engineering, 3, 602-608, 2011.

Antoniou, A. A. and Lekkas, E.: Rockfall susceptibility map for Athinios port, Santorini Island, Greece, Geomorphology, 118, 152-166, 2010.

Assessorato Regionale per i rifiuti e le acque - settore Osservatorio delle Acque: Map of isohyets for the period 1921-2003, available at: http://www.osservatorioacque.it/dati/cartepluviotermo/ isoiete_1921-2003.pdf (last access: 31 October 2013), 2004.

Asteriou, P., Saroglou, H., and Tsiambaos, G.: Determination of geotechnical properties of geological formations for rockfall modeling, Int. J. Rock Mech. Min. Sci., 54, 103-113, 2012.

Atzori, P., Cirrincione, R., Kern, H., Mazzoleni, P., Pezzino, A., Puglisi, G., Punturo, R., and Trombetta, A.: The abundance of 53 elements and petrovolumetric models of the crust in northeastern Peloritani Mountains (Site 8), in: The Abundance of 55 Elements and Petrovolumetric Models in Nine Type Areas from the Crystalline Basements of Italy, with some Geophysical and Petrophysical Data, edited by: Sassi, F. P., Acc. Naz. Sci. XL, 32, 309-358, 2003.

AVI Project: Progetto AVI (Aree vulnerate da calamità idrogeologiche), Regione Sicilia Relazione Finale ed Allegati, CNRGNDCI, http://avi.gndci.cnr.it/, Italy, 1998.

Azzoni, A., La Barbera, G., and Zaninetti, A.: Analysis and prediction of rockfalls using a mathematical model, Int. J. Rock Mech. Min. Sci. Geomech. Abstr., 32, 709-724, 1995.

Bacilieri, C.: I borghi più belli d'Italia, Il fascino dell'Italia nascosta, Società Editrice Romana, Italy, 2012.

Barbano, M. S., Pappalardo, G., Pirrotta, C., and Mineo, S.: Landslide triggers along volcanic rock slopes in eastern Sicily (Italy), Nat. Hazards, 73, 1587-1607, doi:10.1007/s11069-014-1160-1, 2014.

Bieniawski, Z. T.: Engineering Rock Mass Classification, John Wiley \& Son, New York, 251 pp., 1989. 
Budetta, P.: Assessment of rockfall risk along roads, Nat. Hazards Earth Syst. Sci., 4, 71-81, doi:10.5194/nhess-4-71-2004, 2004.

Budetta, P.: Rockfall-induced impact force causing a debris flow on a volcanoclastic soil slope: a case study in southern Italy, Nat. Hazards Earth Syst. Sci., 10, 1995-2006, doi:10.5194/nhess-101995-2010, 2010.

Carbone, S., Catalano, S., Lentini, F., and Vinci, G.: Geological map of Taormina Mountains (Peloritani Mts, northeastern Sicily), S.E.L.C.A., Italy, 1994.

Catalano, S., Di Stefano, P., Sulli, A., and Vitale, F. P.: evoluzione paleogeografia e strutturale della Sicilia e dei mari adiacenti, Naturalista sicil., S. IV, Vol. XIX, 3-4, 143-187, 1995.

Chau, K. T., Wong, R. H. C., and Lee, C. F.: Rockfall problems in HongKong and some new experimental results for coefficient of restitution, Int. J. Rock Mech. Min. Sci., 35, 662-663, 1998.

Chau, K. T., Wong, R. H. C., Liu, J., and Lee, C. F.: Rockfall Hazard Analysis for Hong Kong Based on Rockfall Inventory, Rock Mech. Rock Eng., 36, 383-408, 2003.

Cirrincione, R., Fazio, E., Ortolano, G., Pezzino, A., and Punturo, R.: Fault-related rocks: deciphering the structural-metamorphic evolution of an accretionary wedge in a collisional belt, NE Sicily, Int. Geol. Rev., 54, 940-956, 2012.

Corriere, F. and Russo, R.: Procedura per l'individuazione e la valutazione dei sistemi infrastrutturali di trasporto, Regione Siciliana, Piano Territoriale Urbanistico Regionale, Italy, 2003.

Crosta, G. B. and Agliardi, F.: A methodology for physically based rockfall hazard assessment, Nat. Hazards Earth Syst. Sci., 3, 407-422, doi:10.5194/nhess-3-407-2003, 2003.

De Almeida, J. A. and Kullberg, J. C.: Rockfall hazard and risk analysis for Monte da Lua, Sintra, Portugal, Nat. Hazards, 58, 289-310, 2011.

Dorren, L. K. A. and Seijmonsbergen, A. C.: Comparison of three GIS-based models for predicting rockfall runout zones at a regional scale, Geomorphology, 56, 49-64, 2003.

Dorren, L. K. A., Berger, F., and Putters, U. S.: Real-size experiments and 3-D simulation of rockfall on forested and nonforested slopes, Nat. Hazards Earth Syst. Sci., 6, 145-153, doi:10.5194/nhess-6-145-2006, 2006.

Dueé, G.: Etude géologique des Monts Nebrodi (Sicile), Thèse Fac. Sci., 2 voll, 221-169, Paris, 1969.

Ferrara, V. and Pappalardo, G.: Kinematic analysis of rock falls in an urban area: The case of Castelmola hill near Taormina (Sicily, Italy), Geomorphology, 66, 373-383, 2005.

Ferrero, A. M., Migliazza, M., Roncella, R., and Segalini, A.: Rock cliffs hazard analysis based on remote geostructural surveys: The Campione del Garda case study (Lake Garda, Northern Italy), Geomorphology, 125, 457-471, 2011.

Giani, G. P.: Rock Slope Stability Analysis, A. A. Balkema, Rotterdam, the Netherlands, 1992.

Guzzetti, F., Stark, C. P., and Salvati, P.: Evaluation of flood and landslide risk to the population of Italy, Environ. Manage., 36, 15-36, 2005.

Hoek, E.: Rockfall - A Program in Basic for the Analysis of Rockfalls from Slopes, Department of Civil engineering, University of Toronto, Canada, 1987.

Hoek, E. and Bray, J. W.: Rock Slope Engineering, Third Edition, The Institution of Mining and Metallurgy, London, p. 368, 1981.
ISRM: The complete ISRM suggested methods for rock characterization, testing and monitoring: 1974-2006, in: Suggested Methods Prepared by the Commission on Testing Methods, edited by: Ulusay, R. and Hudson, J. A., International Society for Rock Mechanics, Compilation Arranged by the ISRM Turkish National Group, Kozan Ofset, Ankara, Turkey, p. 628, 2007.

Jaboyedoff, M. and Derron, M.-H.: Integrated risk assessment process for landslides, in: Landslide risk management, edited by: Hungr, O., Fell, R., Couture, R., and Eberhardt, E., Taylor and Francis, Balkema, Rotterdam, 776 pp., 2005.

Kliche, C. A.: Rock Slope Stability SME, Littleton, CO, 1999.

La Sicilia, Catania, 27 February, yr LXVIII, n. 57, 2012.

Lentini, F., Carbone, S., and Guarnieri, P.: Collisional and postcollisional tectonics of the Apenninic-Maghrebian orogen (southern Italy), Geological Society of America Special Paper 409, Geological Society of America, Boulder, Colorado, 57-81, 2006.

Marinos, P. and Tsiambaos, G.: Earthquake triggering rockfalls affecting historic monuments and a traditional settlement in Skyros Island, Greece, Proc. of the International Symposium: Landslide risk mitigation and protection of cultural and natural heritage, Kyoto, Japan, 343-346, 2002.

Markland, J. T.: A useful technique for estimating the stability of rock slopes when the rigid wedge sliding type of failure is expected, Imp. Coll. Rock Mech. Res. Rep. 19, Imperial College of Science and Technology, London, 10 pp., 1972.

Massey, C. I., Hodgson, I. F., and Petley, D. N.: A rockfall simulation study for housing development in Gibraltar, 10th IAEG International Congress, Paper number 377, Nottingham, United Kingdom, 2006.

Michoud, C., Derron, M.-H., Horton, P., Jaboyedoff, M., Baillifard, F.-J., Loye, A., Nicolet, P., Pedrazzini, A., and Queyrel, A.: Rockfall hazard and risk assessments along roads at a regional scale: example in Swiss Alps, Nat. Hazards Earth Syst. Sci., 12, 615-629, doi:10.5194/nhess-12-615-2012, 2012.

National Highway Institute: Rockfall Hazard Rating System participant's manual, NHI Course No. 130220, US Dept. of Transportation, Federal Highway Administration, Publication No. FHWA SA-93-057, USA, 1993.

P.A.I.: Piano Stralcio di Bacino per l'Assetto Idrogeologico, Regione Siciliana, http://www.sitr.regione.sicilia.it/pai/ (last access: 3 February 2014), 2006.

Palma, B., Parise, M., Reichenbach, P., and Guzzetti, F.: Rock-fall hazard assessment along a road in the Sorrento Peninsula, Campania, southern Italy, Nat. Hazards, 61, 187-201, 2012.

Pantelidis, L. and Kokkalis, A.: Designing passive rockfall measures based on computer simulation and field experience to enhance highway safety, Int. J. Rock Mech. Min. Sci., 48, 13691375, 2011.

Parise, M.: Landslide hazard zonation of slopes susceptible to rock falls and topples, Nat. Hazards Earth Syst. Sci., 2, 37-49, doi:10.5194/nhess-2-37-2002, 2002.

Paronuzzi, P.: Calculation models for analysing the propagation of rock blocks mobilised by rockfalls, Riv. Ital. Geotecnica, 1987, 145-165, 1987a.

Paronuzzi, P.: Rockfall-induced block propagation on a soil slope, northern Italy, Envirio. Geol., 58, 1451-1466, 2009.

Pfeiffer, T. J. and Bowen, T. D.: Computer simulation of rockfalls, Bull. Assoc. Eng. Geol., 26, 135-146, 1989. 
Pierson, L. A., Davis, S. A., and Van Vickle, R.: Rockfall Hazard Rating System - Implementation Manual, Federal Highway Administration (FHWA), Report FHWA-OR-EG-90-01, US Dep. of Transp., FHWA, Oregon, USA, 1990.

Poretti, I. and De Amicis, M.: An approach for flood hazard modelling and mapping in the medium Valtellina, Nat. Hazards Earth Syst. Sci., 11, 1141-1151, doi:10.5194/nhess-11-11412011, 2011.

Punturo, R., Kern, H., Cirrincione, R., Mazzoleni, P., and Pezzino, A.: P- and S-wave velocities and densities in silicate and calcite rocks from the Peloritani Mountains, Sicily (Italy): The effect of pressure, temperature and the direction of wave propagation, Tectonophysics, 409, 55-72, 2005.

Richards, L. R., Peng, B., and Bell, D. H.: Laboratory and field evaluation of the normal coefficient of restitution for rocks, In: Proceedings of ISRM Regional Symposium Eurock 2001, 47 June 2001, Espoo, 149-155, 2011.

Robotham, M. E., Wang, H., and Walton, G.: Assessment of risk from rockfall from active and abandoned quarry slopes, T. Inst. Min. Metal., 104, A25-A33, 1995.

Rocscience: DIPS, 5.1-Graphical and statistical analysis of orientation data rocscience, Canada, 2004.

Romana, M.: New adjustment ratings for application of Bieniawski classification to slopes, Proc. Int. Symp. on "The role of rock mechanics", Zacatecas, 49-53, 1985.

Romana, M.: Practice of SMR classification for slope appraisal, Proc. 5th Int. Symp. on Landslides, Balkema, Rotterdam, 12271229, 1988.
Romana, M.: SMR Classification, Proc. 7th Int. Congr. on Rock Mech., Balkema, Rotterdam, 955-960, 1991.

Saroglou, H., Marinos, V., Marinos, P., and Tsiambaos, G.: Rockfall hazard and risk assessment: an example from a high promontory at the historical site of Monemvasia, Greece, Nat. Hazards Earth Syst. Sci., 12, 1823-1836, doi:10.5194/nhess-12-18232012, 2012.

Scandone, P., Patacca, E., Rodoicic, R., Ryan, W. B. F., Cita, M. B., Rawason, M., Cherzar, H., Miller, E., Mckenzie, J., and Rossi, S.: Mesozoic and Cenozoic rocks from Malta Escarpment (Central Mediterranean), A.A.P.G. Bull., 65, 1299-1319, 1981.

Schweigl, J., Ferretti, C., and NÖssing, L.: Geotechnical characterization and rockfall simulation of a slope: a practical case study from South Tyrol (Italy), Eng. Geol., 67, 281-296, 2003.

Tempostretto: online newspaper of Messina District: http://ww2.tempostretto.it/news/, last access: 2 March 2012.

Tortorici, L., Tapponnier, P., and Winter, T.: Faulting during the 1783 Calabric earthquake and tectonics of the Messina strait, EOS Trans. Am. Geophys. Union, 67, 1188, 1986.

Turner, A. K. and Schuster, R. L.: Landslides, Investigation and Mitigation, Transportation Research Board, Special Report 247, National Research Council, National Academy Press, Washington, D.C., 673 pp., 1996.

Uribe-Etxebarria, G., Morales, T., Uriarte, J. A., and Ibarra, V.: Rock cut stability assessment in mountainous regions, Environ. Geol., 48, 1002-1013, 2005.

Yoon, W. S., Jeong, U. J., and Kim, J. H.: Kinematic analysis for sliding failure of multi-faced rock slopes, Eng. Geol., 67, 51-61, 2002. 